\title{
Outdoor recognition at a distance by fusing gait and face
}

\author{
Zongyi Liu *, Sudeep Sarkar \\ Computer Science and Engineering, University of South Florida, Tampa, FL 33647, USA
}

Received 19 June 2004; received in revised form 17 November 2005; accepted 19 May 2006

\begin{abstract}
We explore the possibility of using both face and gait in enhancing human recognition at a distance performance in outdoor conditions. Although the individual performance of gait and face based biometrics at a distance under outdoor illumination conditions, walking surface changes, and time variations are poor, we show that recognition performance is significantly enhanced by combination of face and gait. For gait, we present a new recognition scheme that relies on computing distances based on selected, discriminatory, gait stances. Given a gait sequence, covering multiple gait cycles, it identifies the salient stances using a population hidden Markov model (HMM). An averaged representation of the detected silhouettes for these stances are then built using eigenstance shape models. Similarity between two gait sequences is based on the similarities of these averaged representations of the salient stances. This gait recognition strategy, which essentially emphasizes shape over dynamics, significantly outperforms the HumanID Gait Challenge baseline algorithm. For face, which is a mature biometric for which many recognition algorithms exists, we chose the elastic bunch graph matching based face recognition method. This method was found to be the best in the FERET 2000 studies. On a gallery database of 70 individuals and two probe sets: one with 39 individuals taken on the same day and the other with 21 individuals taken at least 3 months apart, results indicate that although the verification rate at $1 \%$ false alarm rate of individual biometrics are low, their combination performs better. Specifically, for data taken on the same day, individual verification rates are $42 \%$ and $40 \%$ for face and gait, respectively, but is $73 \%$ for their combination. Similarly, for the data taken with at least 3 months apart, the verification rates are $48 \%$ and $25 \%$ for face and gait, respectively, but is $60 \%$ for their combination. We also find that the combination of outdoor gait and one outdoor face per person is superior to using two outdoor face probes per person or using two gait probes per person, which can considered to be statistical controls for showing improvement by biometric fusion.
\end{abstract}

(C) 2006 Elsevier B.V. All rights reserved.

Keywords: Gait recognition; Face recognition; Biometrics fusion

\section{Introduction}

Biometrics for outdoor conditions, especially one that can operate at a distance, is a challenging proposition. For some security situations, it is necessary to identify individuals or even short list possible candidates as far away as possible from a sensitive site. Two biometric sources that are available in such situations are face, which is a physical biometric, and gait, which is a behavioral biometric. Face image based biometrics is now a

\footnotetext{
* Corresponding author. Tel.: +1 813974 2113; fax: +1 8139745456.

E-mail addresses: zliu4@csee.usf.edu (Z. Liu), sarkar@csee.usf.edu (S Sarkar).
}

mature technology. Unlike other traditional biometric sources, such as fingerprints, iris, or hand, face does not require direct contact and is easy to acquire. There are now several commercial systems that can be used for face recognition in somewhat controlled indoor situations. However, outdoor recognition from faces is still an open area of research. The 2002 face recognition vendor tests (FRVT 2002) [34], which is presently the most comprehensive and extensive evaluation, show that when comparing a gallery of indoor full frontal images with a probe set of outdoor images, the best verification performance is $54 \%$ at $1 \%$ false alarm rate. This is in contrast to nearly $96 \%$ verification rate at $1 \%$ false alarm for indoor face recognition. 
Like face, gait of a person, as captured in the video of the walking person, is another biometric source that can be acquired in outdoor conditions and from a distance. Recognition of a person from gait has been a recent focus in computer vision $[33,26,43,3,45,42,13,2,25,21,10,38,49]$. To facilitate the objective, quantitative measurement of progress, and the characterization of the properties of gait recognition on a common data set the HumanID Gait Challenge Problem was formulated $[18,19]$. The challenge problem consists of a baseline algorithm, a set of twelve experiments (A through L), and a large data set (1870 sequences, 122 subjects, 1.2 terabytes of data). Based on the reported performance on this dataset and other datasets, three observations can be made regarding gait recognition.

1. Performance on indoor sequences $[49,13,10]$ generally tend to be higher than on outdoor sequences $[10,20,2]$.

2 . When comparing sequences across surface change conditions, such as grass vs. concrete, the performance is low. The identification rates on a gallery set of 71 subjects range from $21 \%$ to $36 \%$, using variety of recognition strategies, ranging from the use of HMM to simple template based matching $[44,47,24,50,18]$.

3. Gait recognition performance drops when comparing sequences taken at different times. When the difference in time between gallery (the pre-stored template) and probe (the input data) is in the order of minutes, the identification performance ranges from $91 \%$ to $95 \%$ $[49,13,10]$, whereas the performances drop to $30-45 \%$ when the differences are in the order of months and days $[25,11,10]$ for similar sized datasets.

It is unlikely that gait recognition performance across these conditions can be improved significantly by simple modifications of existing strategies. From a computer vision perspective, gait signal is comprised of both shape and dynamics information. Gait shape can be defined to be the configuration the person makes; it is determined by both body shape and stance. Gait dynamics determines the nature of the transition between stances. In traditional gait characterization, the latter is emphasized, while some recent recognition from gait studies seem to point to the observation that shape is a better cue than dynamics. First, is the CMU study [10] that explicitly ignores dynamics and just considers silhouette shapes with improved performance. Second, is the UMD study [48] that decouple shape and dynamics using rigorous shape modeling. One hypothesis for this observation could be that the variability of gait dynamics under different conditions, such as walking surface change, is high. Building on this recent observation in gait recognition research that shape is a better cue than dynamics for gait recognition, we present a new strategy that focuses on stance shapes. The strategy relies on computing distances based on selected, discriminatory, gait stances. Given a gait sequence, covering multiple gait cycles, it identifies the salient stances using a population hidden Markov model (HMM). An averaged representa- tion of the detected silhouettes for these stances are then built using eigenstance shape models. Similarity between two gait sequences is based on the similarities of these averaged representations of the salient stances. It essentially uses gait stances that are more robust across the covariates for the similarity computation. This results in improved performance, but still does not achieve high recognition rates comparable to indoor scenarios.

It has been demonstrated that combination or fusion of biometrics can offer a way to break the barrier of poor individual biometric performance. Lin et al. [14] demonstrate that multi-biometric integration does indeed result in a consistent performance improvement. Schiele [40] empirically showed that the more classifiers we combine, the better results we can get. One can talk about inter-modal combination $[39,51,5,41,42,23,16,15]$, e.g. combination of face with iris, and intra-modal combination [1,53,31, $54,17,36,32,55]$, e.g., combination of outputs of two classifiers on the same modality, or the combination of outputs of two different sensors, such as IR and visible $[9,8]$ and visible and 3D [6-8], on the same modality. In Table 1 we summarize the work in computer-vision based multi-modal biometric combination. Fusion can be done at three levels [39]:

1. The feature extraction level, where data from each sensor are: combined to form one feature vector $[12,5]$.

2. The matching score level, where the similarity scores computed by individual classifier are fused $[39,31,17,16,1,51,54,41,42,23]$. The scores from different classifiers are usually first transformed into the same range using linear transformation, polynomial transformation, or logarithm transformation. The normalized scores are then combined using rules, such as sum, product, maximum, and minimum.

3. The decision level where the each classifier makes its own classification and votes for the final decision $[36,15,23,32]$. The popular vote rules include rank sum and majority vote.

In this paper, we show the combination of gait and face can effectively enhance the performance of outdoor biometrics at a distance. We demonstrate this for conditions that are known to be "hard" in face and gait recognition. Experiments also show that cross modal combination of gait and face is superior to the fusion of multiple instances within each modality, which can be considered to sort of statistical control to show improvement by biometric fusion. Gait and face combination studies have been presented by others $[42,41,22]$. However, unlike previous studies that used either indoor data or outdoor data taken on the same day, resulting in high performance of the individual biometrics to begin with, our study involves outdoor data, taken months apart. We show that we can significantly improve recognition at a distance in outdoor conditions and over time, both of which are hard conditions, using biometric fusion. 
Table 1

Inter- and intra-modal biometric fusion

\begin{tabular}{|c|c|c|c|c|c|c|c|c|}
\hline Work & Combination level & Face & Fingerprint & Hand geometry & Iris & Ear & Gait & Speech \\
\hline MSU [39] & Score & $\sqrt{ }$ & $\sqrt{ }$ & $\sqrt{ }$ & & & & \\
\hline MSU [31] & Score & $\sqrt{ } \sqrt{ }$ & & & & & & \\
\hline MSU [17] & Score & & $\sqrt{ } \sqrt{ }$ & & & & & \\
\hline MSU [16] & Score & $\sqrt{ }$ & $\sqrt{ }$ & & & & & $\sqrt{ }$ \\
\hline MSU [15] & Decision & $\sqrt{ }$ & $\sqrt{ }$ & & & & & \\
\hline MSU [36] & Decision & & $\sqrt{ } \sqrt{ }$ & & & & & \\
\hline U. Bern [1] & Score & $\sqrt{ }$ & & & & & & \\
\hline CAS and MSU [51] & Score & $\sqrt{ }$ & & & $\sqrt{ }$ & & & \\
\hline UND and USF [5] & Score & $\sqrt{ }$ & & & & $\sqrt{ }$ & & \\
\hline HK polytechnic [54] & Score & $\sqrt{ }$ & & & & & & \\
\hline MIT $[41,42]$ & Score & $\sqrt{ }$ & & & & & $\sqrt{ }$ & \\
\hline U. of Surrey [23] & Score and Decision & $\sqrt{ }$ & & & & & & $\sqrt{ }$ \\
\hline Rutgers [32] & Score and Decision & $\sqrt{ } \sqrt{ }$ & & & & & & \\
\hline UMD [22] & Score and Decision & $\sqrt{ }$ & & & & & $\sqrt{ }$ & \\
\hline UND [9,6-8] & Decision & $\sqrt{ } \sqrt{ }$ & & & & & & \\
\hline
\end{tabular}

\section{Face recognition algorithm}

The primary focus of this paper is to investigate the power of the face and gait biometric fusion. So, the individual biometric algorithms we used are not necessarily the absolute best that are currently available, but they have performances that are close to the best available ones. They beat their corresponding established baseline algorithms by significant amounts. First, we consider the face recognition algorithm (Fig. 1).

Face recognition is a mature biometric for which many recognition approaches exist. We did not see the necessity for designing yet another approach since the focus of this paper is biometric fusion. From among the many possible choices, we used the Gabor features based Elastic Bunch Graph Matching (EBGM) [52] algorithm for face recognition. It is a feature based method for face recognition that has superior performance than other template based methods, such as PCA, LDA, or Bayesian. We used the CSU implementation of the algorithms that is available at http://www.cs.colostate.edu/evalfacerec/. The approach first locates landmarks on a face, related to salient points on eyes, nose, and mouth, and then employs the frequency information of the local regions that surround the landmark locations as the landmark features (landmark jet). We did not re-train the algorithm. Instead we used the CSU trained version, which is based on 70 subjects. With regard to distance measurements, we choose the phase similarity, corrected by small displacements [4]:
$S_{D}\left(J_{i}, J_{i}^{\prime}, \vec{d}\right)=\frac{\sum_{j=0}^{N_{i}} a_{i j} a_{i j}^{\prime} \cos \left(\phi_{i j}-\left(\phi_{i j}^{\prime}+\vec{d} \overrightarrow{k_{i j}}\right)\right)}{\sqrt{\sum_{j=0}^{N_{i}} a_{i j}^{2} \sum_{j=0}^{N_{i}} a_{i j}^{\prime 2}}}$

where $J_{j}$ and $J_{i}^{\prime}$ are the landmark jets of $i$ th landmark point for graph $J$ and $J^{\prime}, N_{i}$ is the number of wavelet coefficients in the jet, $a$ and $\phi$ are the magnitude and phase, $\vec{d}$ is the estimated displacement vector, and $\vec{k}$ is a vector pointing in the direction of the wave and having the magnitude equal to frequency of the wave. Obviously, the estimation of the displacement vector $\vec{k}$ is very important for Eq. (1). In this paper, we use the Displacement Estimation Narrowing Local Search (DENarrowingLocalSearch), which uses a local search method to find an optimum and empirically gives the best performance.

According to the FERET evaluations [35], the EBGM approaches provides the best recognition performance. Fig. 2 summarizes the reported top rank identification performance (with a gallery size of 1200) on three experiments involving matching (i) across indoor illumination variations, (ii) across 1 year time differences, and (iii) across more than 1 year time difference. EGBM had the top rank among five algorithms, for all the three experiments. It outperformed by around $20 \%$ the next best algorithm. Also, note the poor performance on datasets that involve comparison over time.
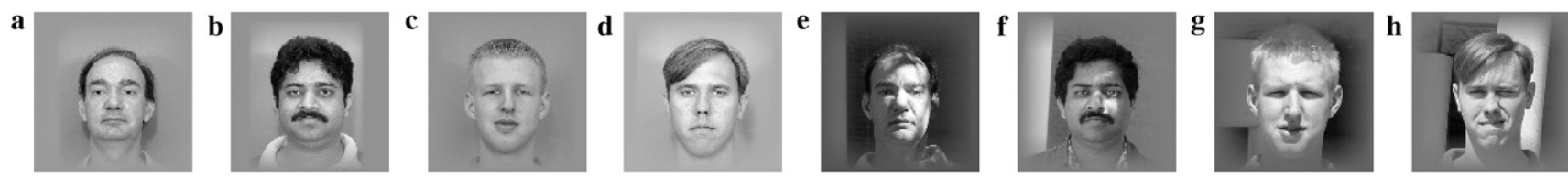

Fig. 1. Samples of computed intermediate representations face biometric that are matched. (a-d) gallery, (e-h) probes. 


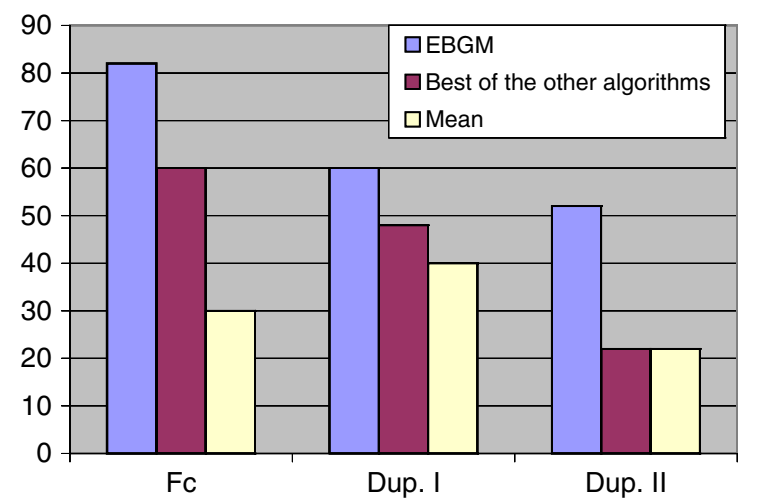

Fig. 2. Top rank identification performance (on a gallery set of 1200) the EBGM and four other face recognition algorithms as reported by FERET-2000 [35]. The experiment $F_{c}$ matches across illumination variation, the Dup. I experiment involves temporal difference within 1 year, and the Dup. II experiment involves temporal difference more than 1 year.

\section{Gait recognition algorithm}

Compared with face, gait biometrics is a relatively new research area. New approaches are beginning to emerge but with varying performances. Fusion of face with gait will make sense only if the performance of these two biometrics are comparable, especially on the hard cases that we consider. For this reason, we designed a new gait recognition algorithm, that is among the best that is currently available, as judged based on commonly available benchmarks.

The recognition approach builds on the observation in recent gait recognition experiments $[48,10]$ that silhouette shape, which includes body shape and gait stance shape, has equal, if not more, recognition power than gait dynamics. The approach is based on matching silhouettes from selected gait stances, specifically those stances that have largest variance across a population. The schematic of the gait recognition approach is shown in Fig. 3. The first step is, of course, silhouette detection, for which we use standard statistical background subtraction, based on Mahalanobis distances in the pixel color space. Second, is the definition and modeling of the stances to be used for recognition. For this, we conduct analysis by learning one population EigenStance-HMM, defined for a subject population. The variation in shape for each stance will help characterize the importance of that stance. Third, is the recognition approach. Any given gait sequence is decoded using the learnt population HMM. The frames that are matched to the selected stances are cleaned-up and averaged to arrive at an averaged silhouette representation for that stance. The averaged stance representation from the gallery and the probe are then compared using the sum of the Euclidean distances between the selected stance silhouettes. In the rest of this section, we present details regarding the above the steps.

\subsection{Silhouette detection}

We employ the standard RGB based background differencing technique to segment silhouettes. We first compute the statistics of the individual background pixels in terms of mean and covariance of RGB values. Then we compute the Mahalanobis distance of a pixel from this background pixel value distribution. Next, we decide on an optimal threshold to segregate the two classes using Expectation Maximization (EM), with the distance values as the Gauss-

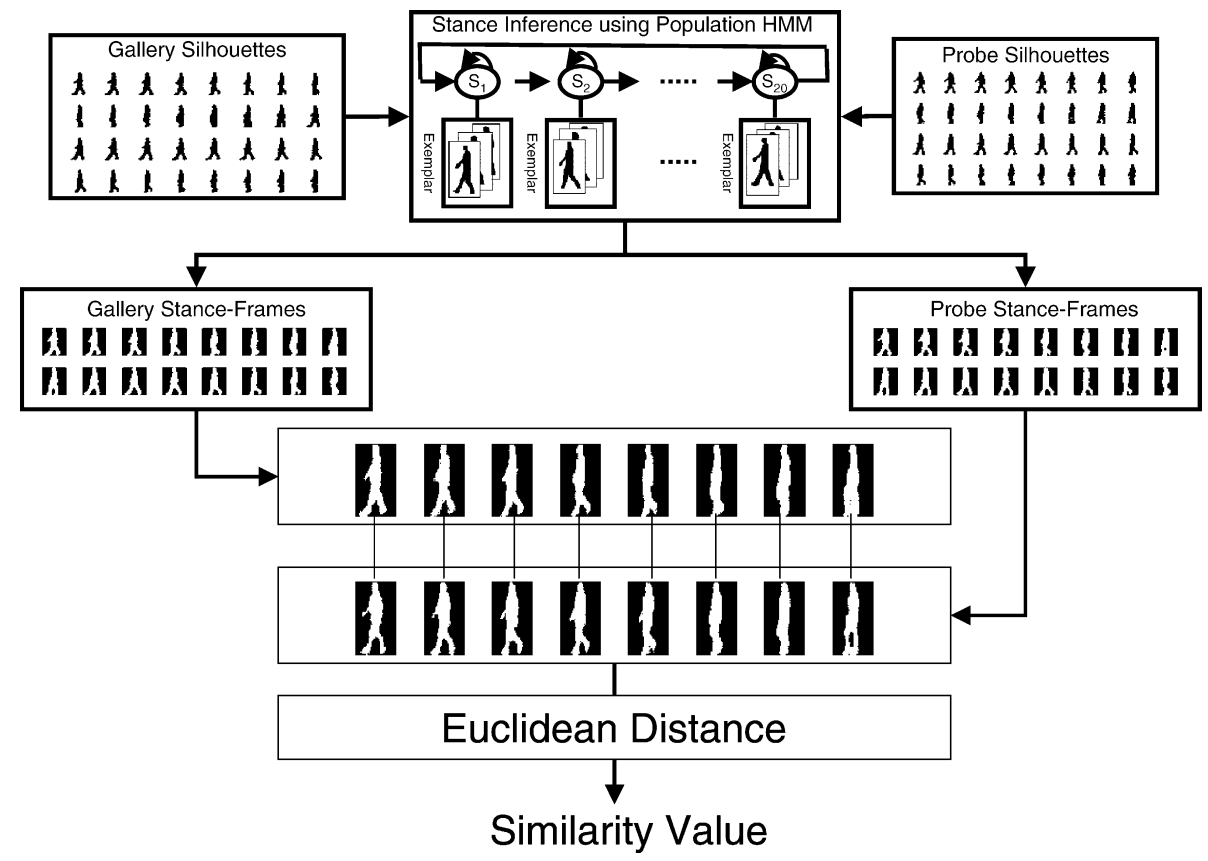

Fig. 3. The adopted gait recognition approach based on comparing specific, salient, gait stances. 
ian distributed observations. Finally, we assemble disconnected components with a proximity based grouping process, using the area and angle features of each component. Further details of this process are available in $[28,30]$.

\subsection{Stance modeling and selection using population $H M M$}

To identify the gait stances that have recognition power, we need to be able to define the different gait stances. Given that gait can be viewed as involving periodic state transition, we use a Hidden Markov Model (HMM) to define and to identify the underlying stances. A HMM is specified by the possible states, $q_{t} \in\left\{1, \ldots, N_{\mathrm{s}}\right\}$, and the triple $\lambda=(A, B, \pi)$, representing the state transition matrix, observation model, and priors, respectively. The states of the HMM are the different gait stances. The state transition matrix and the priors capture the gait dynamics and the observation model is based on the stance shapes. These parameters should capture the variation across a subject population. Note that we do not seek a HMM for each subject, but one HMM defined over the whole population, i.e. a population HMM. The trained or learnt parameters of this model will help identify the underlying stances. This model will also used in the similarity computation step.

\subsubsection{Training of the $H M M$}

The gait stance states and state transition probabilities of this model are learnt from a set of manually specified sil-
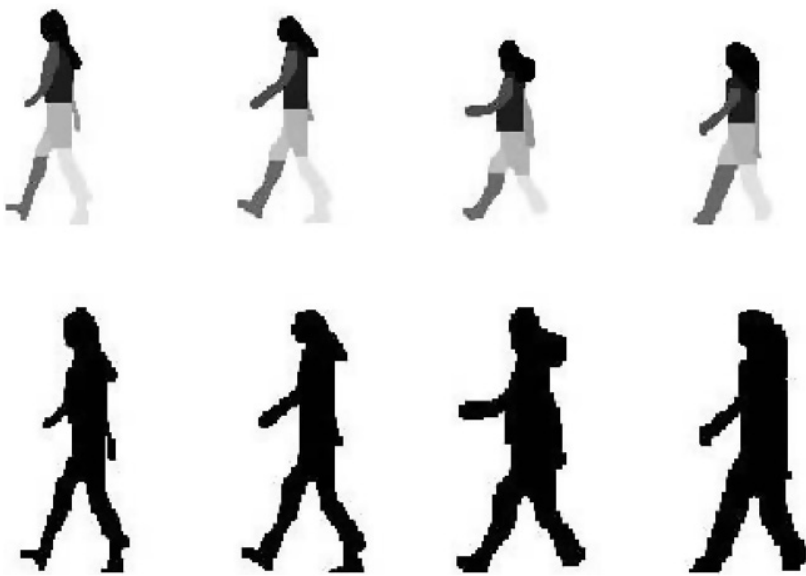

Fig. 4. Top row shows the corresponding part-level, manually specified silhouettes. And the bottom row shows the scaled silhouettes of the kind used by gait recognition algorithms. houettes, which can be taken to be the best "clean" silhouettes that are available. This silhouette database includes 70 subjects over one walking cycle of approximately 30 to 40 image frames [27]. This cycle was chosen to begin at the right heel strike phase of the walking cycle through to the next right heel strike. The height of the silhouettes are normalized to occupy 128 pixels in order to reduce the effect of varying distance of subjects from camera. Fig. 4 shows examples of the raw images and the normalized silhouettes of one subject.

We consider distinct states, $\left(q_{1}, q_{2}, \ldots, q_{N_{\mathrm{s}}}\right)$, spanning one full cycle (two strides) so as to retain the asymmetry in gait, i.e. to differentiate stances with left foot forward from those with right foot forward. We will select the total number of states, $N_{s}$ by minimizing the Akaikie's information criterion (AIC). There are three sets of parameters to be estimated. First, we pick equal state priors, i.e. $\pi_{i}=\frac{1}{N_{\mathrm{s}}}$, since, in practice, any given sequence can begin from any state.

Second, is the observation model, which we base upon state exemplars selected from the training set sequences. These state exemplars are determined as follows. We start with a linear map of the silhouettes in a sequence into the states, which are then refined by K-Means clustering. The clustering technique relies a distance measurement for two frames $f_{i}$ and $f_{j}$, which we define as:

$D(i, j)=1-\frac{\mathbf{f}_{\mathbf{i}}^{T} \mathbf{f}_{\mathbf{j}}}{\mathbf{f}_{\mathbf{i}}^{T} \mathbf{f}_{\mathbf{i}}+\mathbf{f}_{\mathbf{j}}^{T} \mathbf{f}_{\mathbf{j}}-\mathbf{f}_{\mathbf{i}}^{T} \mathbf{f}_{\mathbf{j}}}$

This is also commonly known as the Tanimoto distance measure. Since the gait cycles of the manual silhouettes are aligned by design, we simply group the frames within the $j$ th partition of all subjects into an exemplar set for the $j$ th gait stance, $E_{j}$. Fig. 5 shows the mean images of the exemplars for some stances, built from our training data. The observation model for each stance is chosen to be an exponential function of the Tanimoto distance, $D$, between any given silhouette, $\mathbf{f}_{\mathbf{t}}$, to the mean of the state exemplars, $\bar{E}_{j}$, where the parameter $\mu_{j}$ is directly estimated from the corresponding exemplars which have been computed from the given training sequences.

$b_{j}\left(\mathbf{f}_{\mathbf{t}}\right)=\frac{1}{\mu_{j}} \mathrm{e}^{-\frac{D\left(\mathbf{f}_{\mathbf{f}}, \bar{E}_{j}\right)}{\mu_{j}}}$

The third set of parameters comprise the state transition matrix A. The transition matrix is constrained to be a left to right, cyclical Bakis state transition model over the
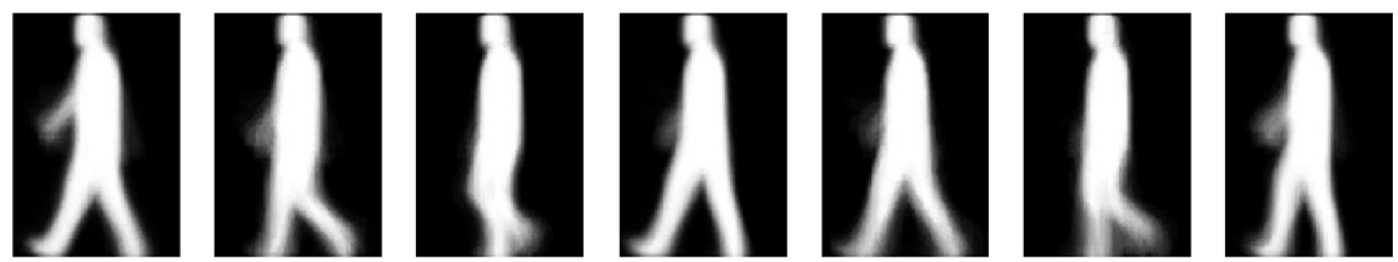

Fig. 5. Stance exemplars for seven sample states over a gait cycle used in the observation model of the population HMM. 
states. We estimate this matrix from the multiple observation sequences using the iterative Baum-Welch algorithm [37]. However, unlike the traditional use of this algorithm, the priors and the observation models are not iterated upon. Just the entries of the transition matrix are iteratively updated to maximize the likelihood of the training set. This initialization of this iterative process is based on the transitions implied by the association of frames to each stance arrived by K-means clustering used to arrive at the observation model. Note that since the training set consists of gait sequences from a number of subjects, the transition matrix represents the average gait dynamics over the whole population.

We determine the number of states based on the Akaike's information criterion (AIC) that combines the likelihood of the data with model complexity in a probabilistically meaningful manner. Fig. 6 shows the AIC variation for different number of states. Small values of $N_{\mathrm{s}}$ would result in a compact model but would not capture all the variations in the training set. Whereas very large values would results in over fitting. We see that $N_{\mathrm{s}}=20$ offers a good compromise. Given the context of gait recognition, we would like to preserve variations so as to be able to distinguish between individuals. We also found that this choice is quite stable with respect to some variation of

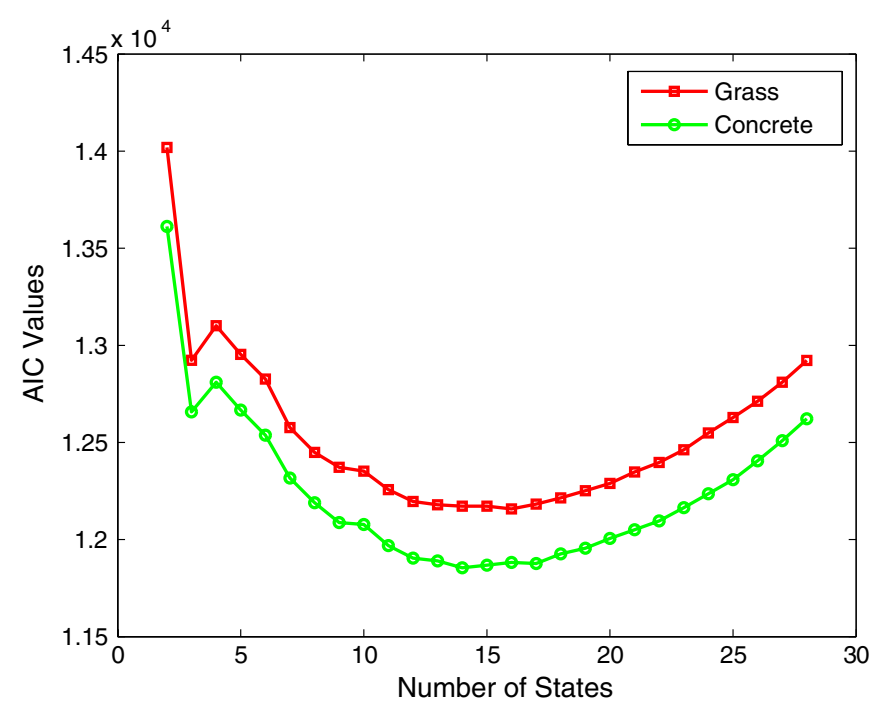

Fig. 6. Variation of AIC with number of states, for models constructed using two different training sets of 70 subjects; one for grass walking surface and the other for concrete walking surface. training set. Fig. 6 shows AIC variation for two different training sets, one based on sequences on grass and other for sequences on concrete.

\subsubsection{Gait shape - eigenstances}

With each stance of the trained population HMM, we associate an eigenstance model to capture the shape variations in the silhouettes for each stance across persons. We use this model to compute similarity between two gait sequences. We model the shape as a multivariate Gaussian distribution, which is estimated from the clustered set of exemplar silhouettes associated with each HMM stance. Note that the exemplar means were used to construct the observation model in the HMM. We use the variance for shape modeling. We use principal component analysis (PCA) to arrive at a compact representation of this distribution. For each stance, $k$, we have a reduced dimensional (with $N_{\mathrm{e}}$ dimensions) shape space, $\phi(k)$, characterized by the mean, $\mu_{\mathbf{k}}$ and the eigenvectors $\left\{\mathbf{e}_{\mathbf{k}, 1}, \ldots, \mathbf{e}_{\mathbf{k}, \mathbf{N}_{e}}\right\}$. The sample eigenstances representing the most discriminating directions among persons are shown in Fig. 7. The number of eigenvectors, $N_{\mathrm{e}}$, is chosen so that at least $80 \%$ of the variation is modeled. In another work [30], we had used the EigenState-HMM model to clean up shadow artifacts in silhouettes. In this work, we build our recognition strategy around it.

\subsubsection{Stance selection}

For recognition, we are interested the stances that offer the most discrimination between subjects. To select these discriminatory stances, we consider the variation in shape for each stance as reflected in the first and the second eigenvalues associated with the corresponding Eigenstance model. These are plotted in Fig. 8. We see that states at the ends (states 1-3 and 18-20) and at the middle (9-12) has the largest scatters, indicating that these gait stances carry the bulk of the discriminatory power. The mean stances for these states are shown in Fig. 9 for illustration. We notice that these stances correspond to near the full stride stances.

\subsection{Similarity computation}

We based the similarity computation between any two gait sequences on the differences in silhouette shapes of the discriminative stances, as identified during the construction of the eigenstance models. The first step in this
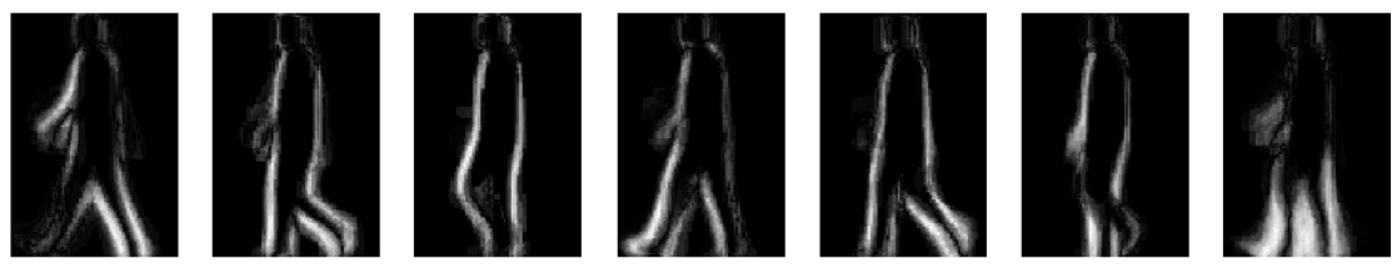

Fig. 7. Samples of the first eigenstances over one gait cycle, representing the most discriminating directions among persons. 


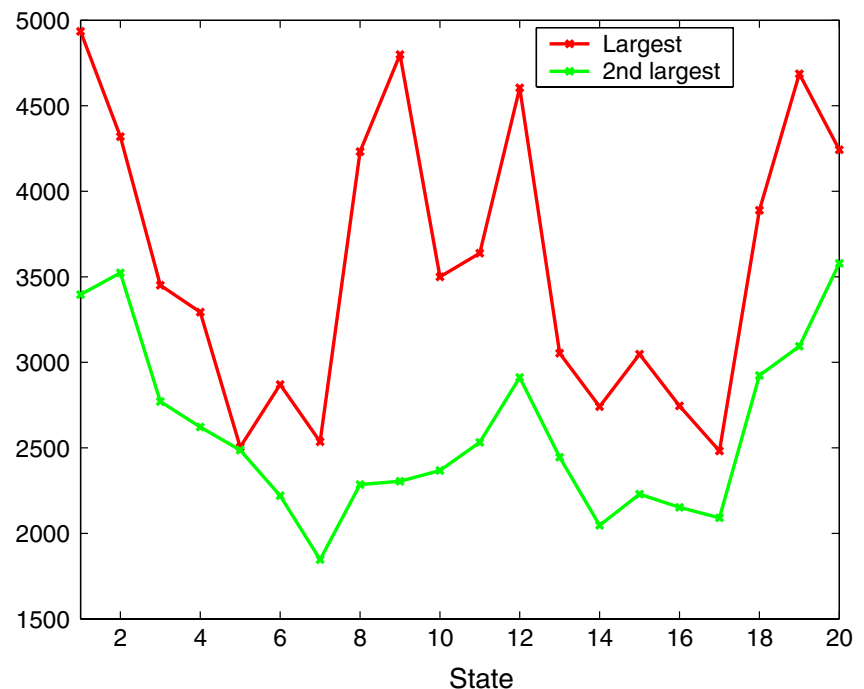

Fig. 8. The variation of largest and second largest eigenvalues associated with each stance shape, as computed in the eigenstance model.

process is the detection of the discriminative stances in any given gait sequence spanning multiple gait cycles. For this, we use the learnt population HMM. We use the dynamic programming based Viterbi algorithm [37], which returns the most likely state assignment for each frame in the given sequence. To reduce the errors of this decoding process, we partition the input sequence into subsequences of roughly one gait cycle length. If frames in one portion of the gait cycle have segmentation errors, this strategy minimizes the effect the stance assignment on other parts of the sequence. We estimate the gait cycle length from the periodic variation in the number of foreground pixels in the bottom half of the silhouettes. Note that the starting state of these subsequences need not match the starting HMM state since we use a cyclical Bakis model for the HMM state transition.

Given the stance labels of each frame, we average the frames mapped to each discriminative stance, identified earlier, to arrive at one averaged representation per stance. However, instead of averaging the raw silhouettes that contain errors due to missed detection and shadows. We use the eigenstance shape model to clean up the silhouettes before averaging.

An input frame $\mathbf{f}_{\mathbf{i}}$, estimated to be at stance state $k$ of the HMM, is projected into the corresponding eigenspace, $\phi(k)=\left\{\mu_{\mathbf{k}}, \mathbf{e}_{\mathbf{k}, \mathbf{1}}, \ldots, \mathbf{e}_{\mathbf{k}, \mathbf{N}_{e}}\right\}$, and then reconstructed as $\mathbf{f}_{\mathbf{i}}^{\mathbf{r}}$

$\mathbf{f}_{\mathbf{i}}^{\mathbf{r}}=\mu_{\mathbf{k}}+\sum_{j=1}^{N_{\mathrm{e}}}\left(\mathbf{e}_{\mathbf{k}, \mathbf{j}}^{T}\left(\mathbf{f}_{i}-\mu_{\mathbf{k}}\right)\right) \mathbf{e}_{\mathbf{k}, \mathbf{j}}$

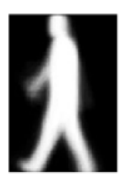

1

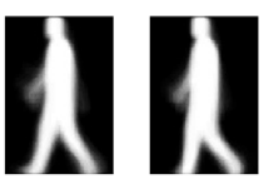

3

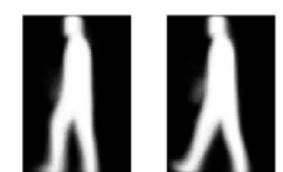

9
10
The reconstructed silhouette, $\mathbf{f}_{\mathbf{i}}^{r}$, has continuous values between 0 and 1 that we threshold to arrive at binary silhouettes. Instead of simple thresholding, we employ a two-level thresholding scheme to minimize the side effect of reconstruction process, which can make silhouettes more similar to each other.

$$
\mathbf{F}_{\mathbf{i}}^{\mathbf{r}}(j)=\left\{\begin{array}{ll}
\text { Foreground } & \text { if } \mathbf{f}_{\mathbf{i}}^{\mathbf{r}}(j)>T_{\text {high }} \text { or } \mu_{\mathbf{k}}(j)=0 \\
\text { Background } & \text { if } \mathbf{f}_{\mathbf{i}}^{\mathbf{r}}(j)<T_{\text {low }} \\
\mathbf{f}_{\mathbf{i}}(j) & \text { otherwise. }
\end{array}\right\}
$$

For the experiments in this paper, $T_{\text {low }}=0.2$ and $T_{\text {high }}=0.8$. Fig. 10 show some averaged stance shape representations constructed for a subject based on a given input sequence.

Given two averaged stance representation, the corresponding stances can be simply compared and the results summed to arrive at an overall similarity score. Let us denote the subset of salient discriminatory states by $\mathbf{S}_{\mathbf{d}}$. To arrive at one similarity score, we compute Euclidean distances between the averaged representation for these stances from the probe sequence $I_{P_{i}}$ and the gallery sequence $I_{G_{j}}$.

$$
S\left(I_{P_{i}}, I_{G_{j}}\right)=-\sum_{k \in \mathbf{S}_{\mathbf{d}}}\left(I_{P_{i}}\left(f_{k}\right)-I_{G_{j}}\left(f_{k}\right)\right)^{2}
$$

\subsection{Performance}

The selected stance based recognition scheme performs well in practice. Table 2 reports top rank identification rate for two key experiments, comparing sequences across surface and across time, from the HumanID Gait Challenge problem $[18,19]$. These experiments, denoted by $\mathrm{D}$ and $\mathrm{K}$ in the original problem, are among the toughest experiments on which most algorithms have poor performance. The gallery set consists of sequences from 122 subjects. The first column of Table 2 lists the performances for the baseline algorithm defined along with the Gait Challenge. We also report performances of four other gait recognition algorithms based on hidden Markov models, shape clustering, part features, and template matching (note that since the individual groups are yet to publish the results officially, we cannot identify them at this time). In the last two columns, we list the performance of the our gait recognition algorithm with and without stance selection. We see that stance selection greatly enhances performance. Note that the proposed gait recognition algorithm's performance is the second best among the six algorithms listed.

Fig. 9. Exemplar means for the states exhibiting significant recognition power. The numbers below each frame denote the state. 

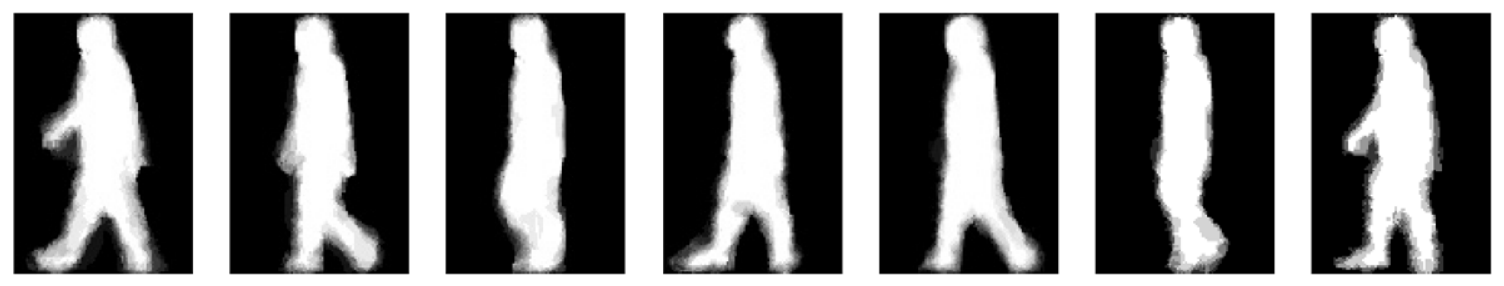

Fig. 10. Averaged gait stances for one subject computed from a sequence spanning several gait cycles.

Table 2

The top rank identification rate for different gait recognition approaches on the gait challenge experiments involving the "hard" covariates of surface and time

\begin{tabular}{llllllll}
\hline Covariates & Baseline & Algol & Algo2 & Algo3 & Algo4 & Average gait stance (all states) & Average gait stance (partial states) \\
\hline$(\operatorname{Exp} D)$ surface & 32 & 33 & 45 & 19 & 23 & 25 & 38 \\
$(\operatorname{Exp} K)$ time & 3 & 15 & 24 & 3 & 6 & 24 & 24 \\
\hline
\end{tabular}

The gallery size is 122 subjects.

\section{Fusion schemes}

Before combination, scores from each classifier are transformed to a common range. Here, we choose the Gaussian model based $z$-normalization, which was also used in FRVT-2002 [34]. For a given probe $p$, we compute its similarity values with all subjects in the gallery set $\left(g_{1}, g_{2}, \ldots, g_{N_{\mathrm{G}}}\right)$. Then we compute the mean value $\left(\mu_{p}\right)$ and standard deviation $\left(\sigma_{p}\right)$ of the similarity values. The similarity value between each $p$ and $G_{j}$ is normalized as:

$\operatorname{NormSim}\left(p, g_{j}\right)=\frac{\operatorname{Sim}\left(p, g_{j}\right)-\mu_{p}}{\sigma_{p}}$

This normalization not only maps the score onto a common scale, but also removes the dependencies of the scores on the particular probe. It is common in biometrics to observe that the non-match similarity scores are dependent on the chosen probe. This impacts the optimality of the single threshold decision rule chosen for verification in biometric systems.

We experimented with score level and decision level integration.

1. Score Sum combination strategy makes decision simply based on the sum of the similarity scores from the gait and face classifiers:

$\operatorname{CombSim}\left(p, g_{j}\right)=\operatorname{NormSim}_{1}\left(p, g_{j}\right)+\operatorname{NormSim}_{2}\left(p, g_{j}\right)$

2. The second score fusion scheme is based on the Bayesian decision rule. For a given pair of probe and gallery subjects, the similarity values from the individual modalities form the observation vector, v. The two classes correspond to the match (genuine, $\omega_{\mathrm{m}}$ ) and non-match (imposter, $\omega_{\mathrm{nm}}$ ) classes. The likelihoods of these two classes are modeled as multi-dimensional Gaussian distribution, which is usually a good choice empirically. Fig. 11 shows a 2D histogram representation of the gait and face non-match (imposter) scores.

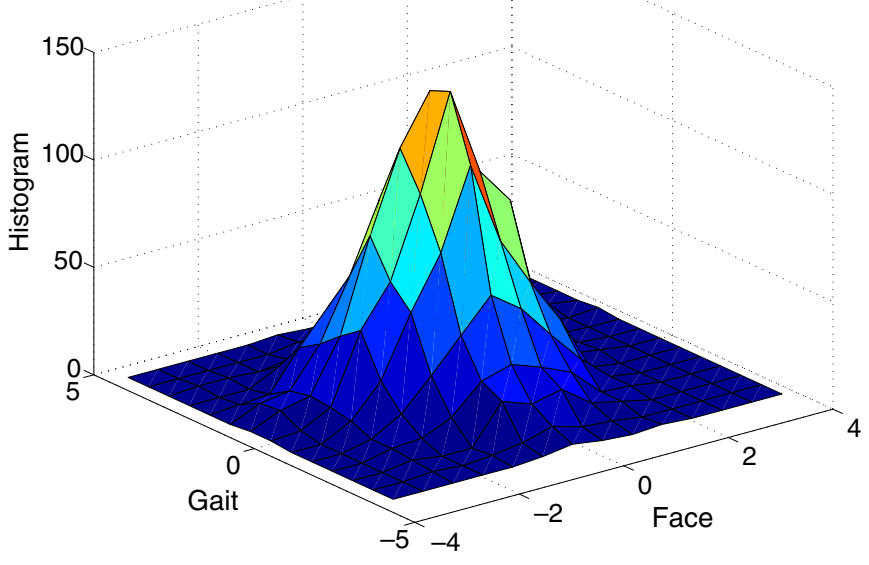

Fig. 11. The 2D histogram of face and gait non-match scores.

$\operatorname{Pr}\left(\mathbf{v} \mid \omega_{\mathrm{m}}\right)=\frac{1}{2 \pi\left|\Sigma_{m}\right|^{1 / 2}} \mathrm{e}^{\left[-\frac{1}{2}\left(\mathbf{v}-\mu_{m}\right)^{T} \Sigma^{-1}\left(\mathbf{v}-\mu_{m}\right)\right]}$
$\operatorname{Pr}\left(\mathbf{v} \mid \omega_{\mathrm{m}}\right)=\frac{1}{2 \pi\left|\Sigma_{m}\right|^{1 / 2}} \mathrm{e}^{\left[-\frac{1}{2}\left(\mathbf{v}-\mu_{m}\right)^{T} \Sigma^{-1}\left(\mathbf{v}-\mu_{m}\right)\right]}$

The difference in the posterior probabilities of these two classes form the combined similarity score.

$\operatorname{CombSim}\left(p, g_{j}\right)=\operatorname{Prob}\left(\omega_{\mathrm{m}} \mid \mathbf{v}\right)-\operatorname{Prob}\left(\omega_{\mathrm{nm}} \mid \mathbf{v}\right)$

3. The third scheme is the Confidence Weighted Score Sum as suggested by the HumanID group at University of Notre Dame. The main idea is that for a given probe subject $p$, we weight its similarity scores in a classifier before combination. The weight is computed from the similarity values of the first few ranks:

$W_{c}(p)=\frac{\operatorname{Sim}_{c}(p)(1)-\operatorname{Sim}_{c}(p)(2)}{\operatorname{Sim}_{c}(p)(2)-\operatorname{Sim}_{c}(p)(3)}$ 
where $\operatorname{Sim}_{c}(p)(k)$ is the $k$ th largest similarity value of $p$ when compared to the entire gallery set. The score combination is then given by:

$$
\operatorname{CombSim}\left(p, g_{j}\right)=W_{1}(p) \operatorname{Sim}_{1}\left(p, g_{j}\right)+W_{2}(p) \operatorname{Sim}_{2}\left(p, g_{j}\right)
$$

4. In addition to the score level combination schemes mentioned above, we also use a decision level combination: Rank Sum. It takes the negated sum of ranks from the face classifier and gait classifier as the similarity value:

$$
\operatorname{CombSim}\left(p, g_{j}\right)=-\operatorname{Rank}_{1}\left(p, g_{j}\right)+\operatorname{Rank}_{2}\left(p, g_{j}\right)
$$

A problem of this scheme is that there might be two or more gallery subjects having a same similarity value with a probe. In this paper, the tie is broken by the sum of the original scores in each classifier.

\section{Results}

We conducted a series of studies geared towards answering the following questions in the context of outdoor biometrics:

1. What is the performance of face + gait combination for the same-day data and months-apart data? How does the combination of face and gait compare with single modality?

2. Which combination scheme performs the best?

3. How does the combination of face and gait compare against using multiple samples of the same modality, i.e. face + face or gait + gait?

Answers to the above questions requires careful specification of multiple gallery and probe sets. For faces, the main gallery set $\left(F_{\mathrm{In}, \mathrm{Mug}, t_{1}}\right)$ consists of 70 faces taken indoors with regular expression and mugshot lighting conditions. The alternate gallery set $\left(F_{\mathrm{In}, \mathrm{Ov}, t_{1}}\right)$ consists of the corresponding faces taken with overhead lighting. The outdoor images form the probes. Fig. 12 shows examples of faces for various lighting conditions. There are four face probe sets, with two probe sets per imaging session. For each imaging session, the near images form one set and the far images form the other set. One pair $\left(F_{\text {Out,Near, } t_{1}}, F_{\text {Out,far }, t_{1}}\right)$ was taken on the same day as the indoors images; there 39 such subjects. And the other pair $\left(F_{\text {Out,Near }, t_{2}}, F_{\text {Out,far }, t_{2}}\right)$ was taken at least 3 months apart; there are 21 such subjects.

For gait, the probes and the gallery are constructed from the HumanID Gait Challenge Dataset [18]. The main gallery $\left(G_{\mathrm{Grass}, R, t_{1}}\right)$ consists of sequences from 70 individuals walking on grass, outdoors, viewed from the right camera. The alternate gallery set $\left(G_{\mathrm{Grass}, R, t_{1}}\right)$ consists of the corresponding sequences taken from the left camera, with a verging angle of approximately $30^{\circ}$ to the right view. Like the face, we consider four different probes. The left and right views of the gait on a different surface condition, i.e. concrete, taking on the same day as the gallery, form two probes $\left(G_{\text {Concrete }, R, t_{1}}, G_{\text {Concrete, } L, t_{1}}\right)$, respectively. Fig. 13 shows some sample views. Like face, we also consider the time covariate and consider two more probe sets $\left(G_{\text {Grass }, R, t_{2}}, G_{\text {Grass }, L, t_{2}}\right)$ taken 6 months apart. The sizes of the probe sets match that for the face to allow us to consider biometric combinations.

Based on these gallery and probe, ten experiments were designed, as shown in Table 3. First five experiments deal with same day data and the next five deal with comparing data taken more than 3 months apart. Each set of five experiments consists of experiments to study face and gait, individually and with inter-modal and intra-modal combinations. We report two kinds of recognition rates: one for the verification scenario ( 1 to 1 matching) and the other for the identification scenario ( 1 to $\mathrm{N}$ matching). For the verification scenario, the performance is specified in terms of standard false alarm and detection rates, plotted as a receiver operating characteristic (ROC). In our experiments, we plot the ROCs based on the Parzen windowed, non-parametric, estimates of the match (genuine) or nonmatch (imposter) distributions based on the given data. For the identification scenario, we report the correct identification rates at rank, $k$, i.e. fraction of times the correct match to a probe (the input data) within the top $k$-ranked match among all the matches of that probe to the complete gallery set (the pre-stored templates or models). The plot of the identification rate with rank is called the Cumulative Match Characteristic (CMC). This is a standard perfor-
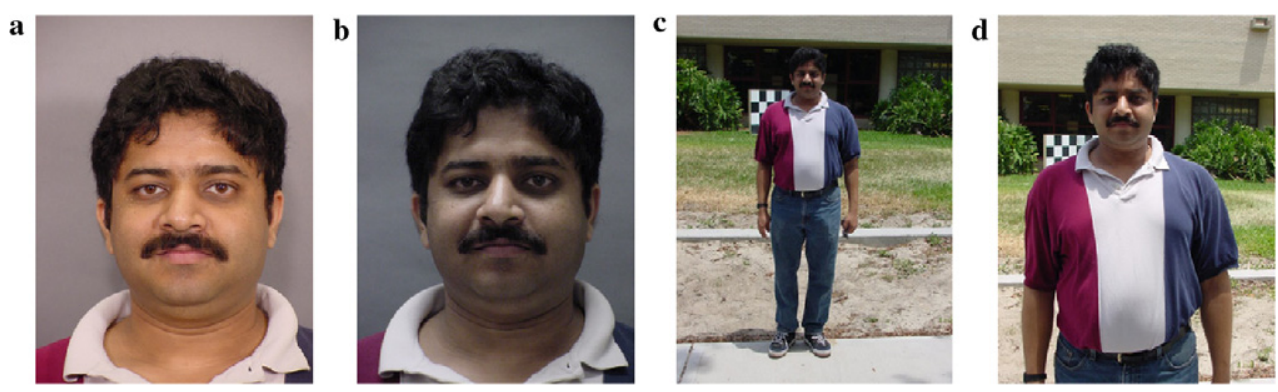

Fig. 12. The face samples under different conditions. The candidates for the gallery sets are (a) regular expression with mugshot lighting, and (b) regular expression, overhead lighting images. The probes are taken outdoors with (c) regular expression, far view and (d) regular expression, near view. 

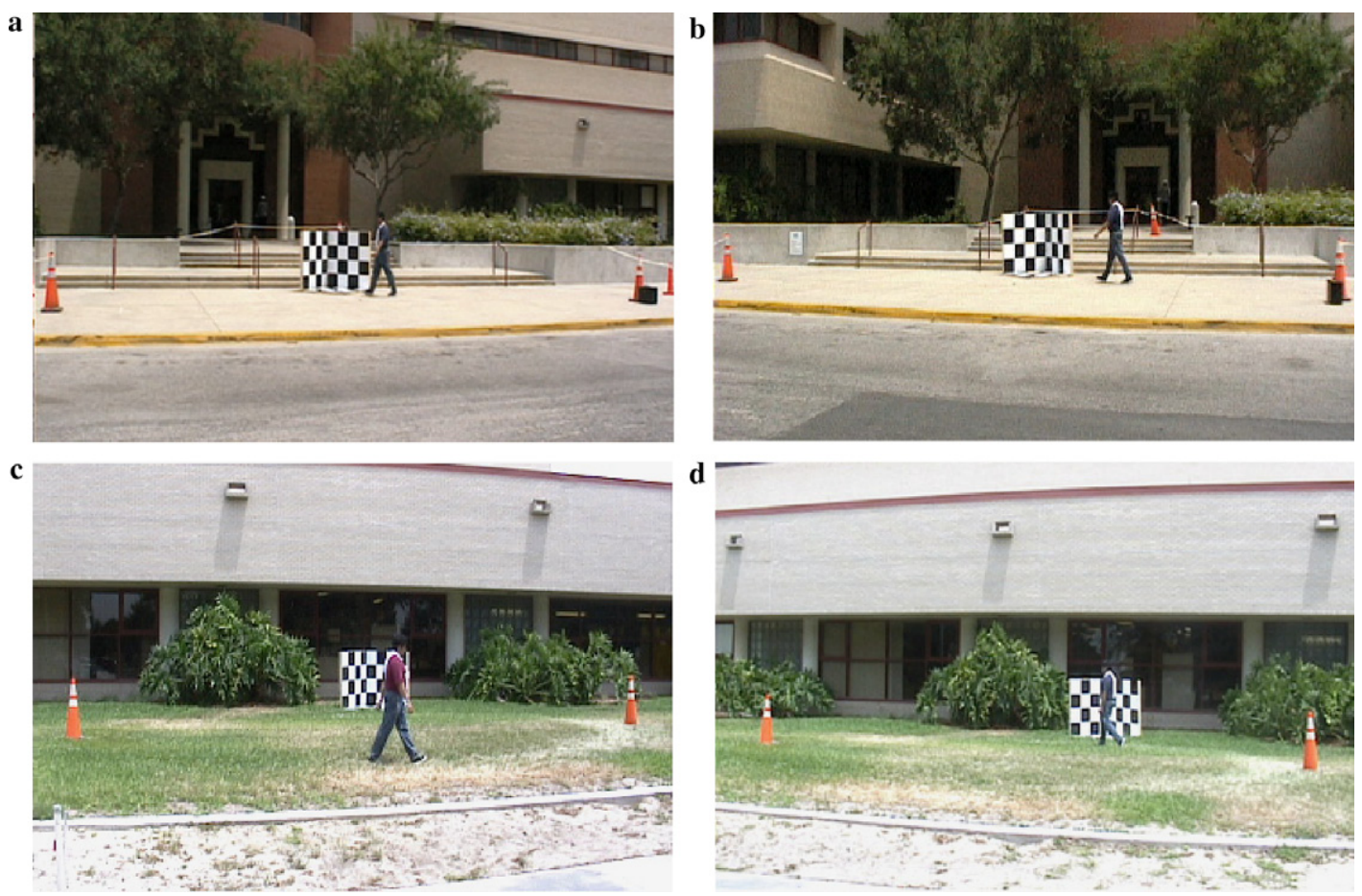

Fig. 13. Sample frames in the gait challenge dataset as viewed from (a) the left camera on concrete surface, (b) the right camera on concrete surface, (c) the left camera on grass surface, (d) the right camera on grass surface.

Table 3

Gallery and probe specification for the various experiments conducted

\begin{tabular}{|c|c|c|c|c|}
\hline Num & Exp & (Gallery, \#) & (Probe, \#) & Covariate \\
\hline$S_{\mathrm{F}}$ & Face & $F_{\ln , \text { Mug }, t_{1}}, 70$ & $F_{\text {Out,near }, t_{1}}, 39$ & In/outdoor, SameDay \\
\hline$S_{\mathrm{G}}$ & Gait & $G_{\mathrm{Grass}, R, t_{1}}, 70$ & $G_{\text {Concrete }, R, t_{1}}, 39$ & Surface, SameDay \\
\hline \multirow{2}{*}{$S_{\mathrm{F}+\mathrm{G}}$} & Face + & $F_{\text {In,Mug }, t_{1}}, 70$ & $F_{\text {Out,near }, t_{1}}, 39$ & In/outdoor, SameDay \\
\hline & Gait & $G_{\mathrm{Grass}, R, t_{1}}, 70$ & $G_{\text {Concrete }, R, t_{1}}, 39$ & Surface, SameDay \\
\hline \multirow[t]{2}{*}{$S_{\mathrm{F}+\mathrm{F}}$} & Face + & $F_{\ln , \text { Mug }, t_{1}}, 70$ & $F_{\text {Out }, \text { near }, t_{1},}, 39$ & In/outdoor, SameDay \\
\hline & Face & $F_{\ln , \mathrm{Ov}, t_{1}}, 70$ & $F_{\text {Out,far, } t_{1}}, 39$ & In/outdoor, SameDay \\
\hline \multirow[t]{2}{*}{$S_{\mathrm{G}+\mathrm{G}}$} & Gait+ & $G_{\mathrm{Grass}, R, t_{1}}, 70$ & $G_{\text {Concrete }, R, t_{1}}, 39$ & Surface, SameDay \\
\hline & Gait & $G_{\text {Grass } L, t_{1}}, 70$ & $G_{\text {Concrete }, L, t_{1}}, 39$ & Surface, SameDay \\
\hline$D_{\mathrm{F}}$ & Face & $F_{\ln , \text { Mug }, t_{1}}, 70$ & $F_{\text {Out,near }, t_{2}} 21$ & In/outdoor, $\geqslant 3$ months apart \\
\hline$D_{\mathrm{G}}$ & Gait & $G_{\mathrm{Grass}, R, t_{1}}, 70$ & $G_{\mathrm{Grass}, R, t_{2}}, 21$ & 6 months apart \\
\hline \multirow[t]{2}{*}{$D_{\mathrm{F}+\mathrm{G}}$} & Face & $F_{\ln , \mathrm{Mug}, t_{1}}, 70$ & $F_{\text {Out,near }, t_{2}}, 21$ & In/outdoor, $\geqslant 3$ months apart \\
\hline & Gait & $G_{\mathrm{Grass}, R, t_{1}}, 70$ & $G_{\mathrm{Grass}, R, t_{2}}, 21$ & 6 months apart \\
\hline \multirow[t]{2}{*}{$D_{\mathrm{F}+\mathrm{F}}$} & Face & $F_{\ln , \text { Mug }, t_{1}}, 70$ & $F_{\text {Out,near }, t_{2}}, 21$ & In/outdoor, $\geqslant 3$ months apart \\
\hline & Face & $F_{\ln , \mathrm{Ov}, t_{1}}, 70$ & $F_{\text {Out,far }, t_{2}}, 21$ & In/outdoor, $\geqslant 3$ months apart \\
\hline \multirow{2}{*}{$D_{\mathrm{G}+\mathrm{G}}$} & Gait+ & $G_{\mathrm{Grass}, R, t_{1}}, 70$ & $G_{\mathrm{Grass}, R, t_{2}}, 21$ & 6 months apart \\
\hline & Gait & $G_{\mathrm{Grass}, L, t_{1}}, 70$ & $G_{\mathrm{Grass}, L, t_{1}}, 21$ & 6 months apart \\
\hline
\end{tabular}

mance metric used in biometrics for the identification scenario [35].

\subsection{Inter-modal combination}

Performance of outdoor face $\left(\operatorname{Exp} S_{\mathrm{F}}\right)$, cross surface gait $\left(\operatorname{Exp} S_{\mathrm{G}}\right)$, and gait + face $\left(\operatorname{Exp} S_{\mathrm{F}+\mathrm{G}}\right)$ on same day data with various combination schemes is shown in Fig. 14, which plots the CMC curve up to rank 5 and ROC curve up to $5 \%$ false alarm rate. As expected, the recognition from a single biometric is low, specifically, $40 \%$ for face and $39 \%$ for gait at rank 1 . However, the combinations of the two weak biometrics using the four schemes discussed above substantially boosts performance. Particularly, $71 \%$ for score sum, $70 \%$ for Bayesian rule, $58 \%$ for confidence weighted score sum, and 68\% for rank sum. As Fig. 15 shows, a similar pattern is seen for performance of outdoor face $\left(\operatorname{Exp} D_{\mathrm{F}}\right)$, cross surface gait $\left(\operatorname{Exp} D_{\mathrm{G}}\right)$, and gait + face $\left(\operatorname{Exp} D_{\mathrm{F}+\mathrm{G}}\right)$ on data taken months apart.

\subsection{Intra-modal combination}

The performance of inter-modal combination has to be justified in the context of intra-modal combination. 

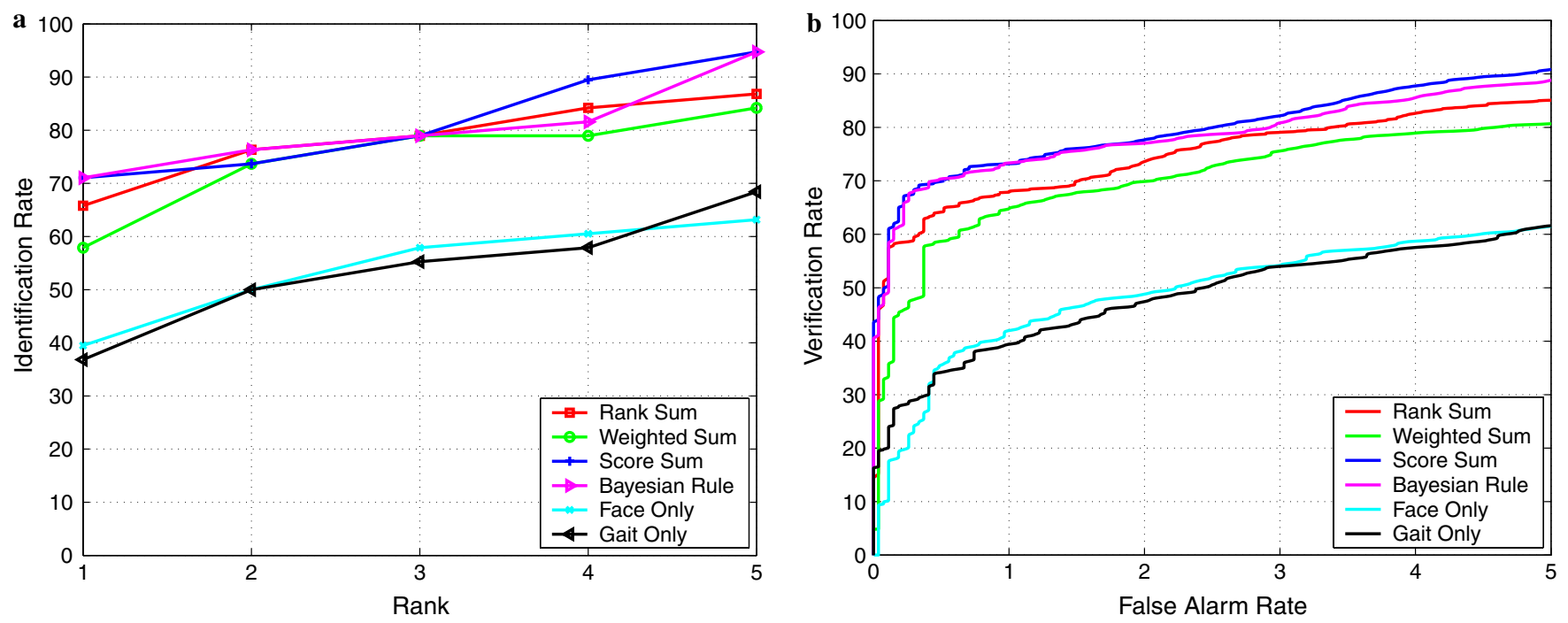

Fig. 14. Performance of outdoor face $\left(\operatorname{Exp} S_{\mathrm{F}}\right)$, cross surface gait $\left(\operatorname{Exp} S_{\mathrm{G}}\right)$, and gait + face $\left(\operatorname{Exp} S_{\mathrm{F}+\mathrm{G}}\right)$ on same day data with various combination schemes for (a) identification and (b) verification scenarios.
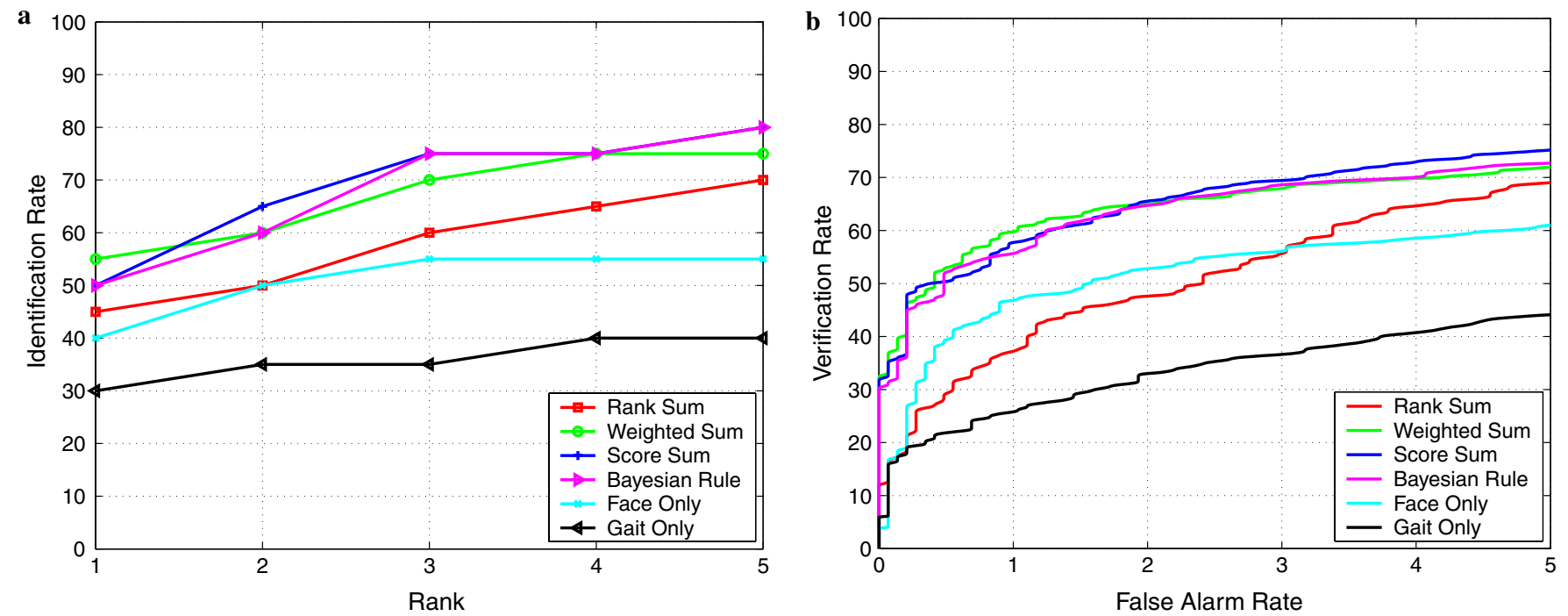

Fig. 15. Performance of outdoor face $\left(\operatorname{Exp} D_{\mathrm{F}}\right)$, cross surface gait $\left(\operatorname{Exp} D_{\mathrm{G}}\right)$, and gait + face $\left(\operatorname{Exp} D_{\mathrm{F}+\mathrm{G}}\right)$ on data taken months apart with various combination schemes for (a) identification and (b) verification scenarios.

Inter-modal combinations involves the use of different types of sensors resulting in added integration costs. The inter-modal performance gain has to be justified in this context. Inter-modal combination performance has to be greater than intra-modal combination [Kevin Bowyer, personal communication]. In the present context, performance of gait and face should be greater than combination of two faces or combination of two gait signatures. For this, we consider the experiments, $S_{\mathrm{F}+\mathrm{F}}, S_{\mathrm{G}+\mathrm{G}}, D_{\mathrm{F}+\mathrm{F}}$, and $D_{\mathrm{G}+\mathrm{G}}$, in Table 3.

These intra-modal experiments involve the use of two samples per subject in the gallery and in the probe. Each probe is matched against the two gallery samples per person and the maximum similarity score is chosen as the sim- ilarity score for that probe. These similarity scores are then combined, as before, using the rules described in Section 4.

Fig. 16 plots the ROCs of the intra-modal combinations up to a false alarm rate of $5 \%$. Each plot shows the performance with individual probes and their combinations. We see that the intra-modal combination does not seem to improve performance by a significant amount. Fig. 17 shows a summary comparison of the inter-modal and intra-model comparison schemes based on the verification rate at a false alarm rate of $5 \%$. We see that face + gait performance is better than the face + face or gait + gait combinations. This is explained by the strong correlation that exist between the scores for two probes from the same biometric. It is 0.7 for the intra-modal case and is only 0.05 for 

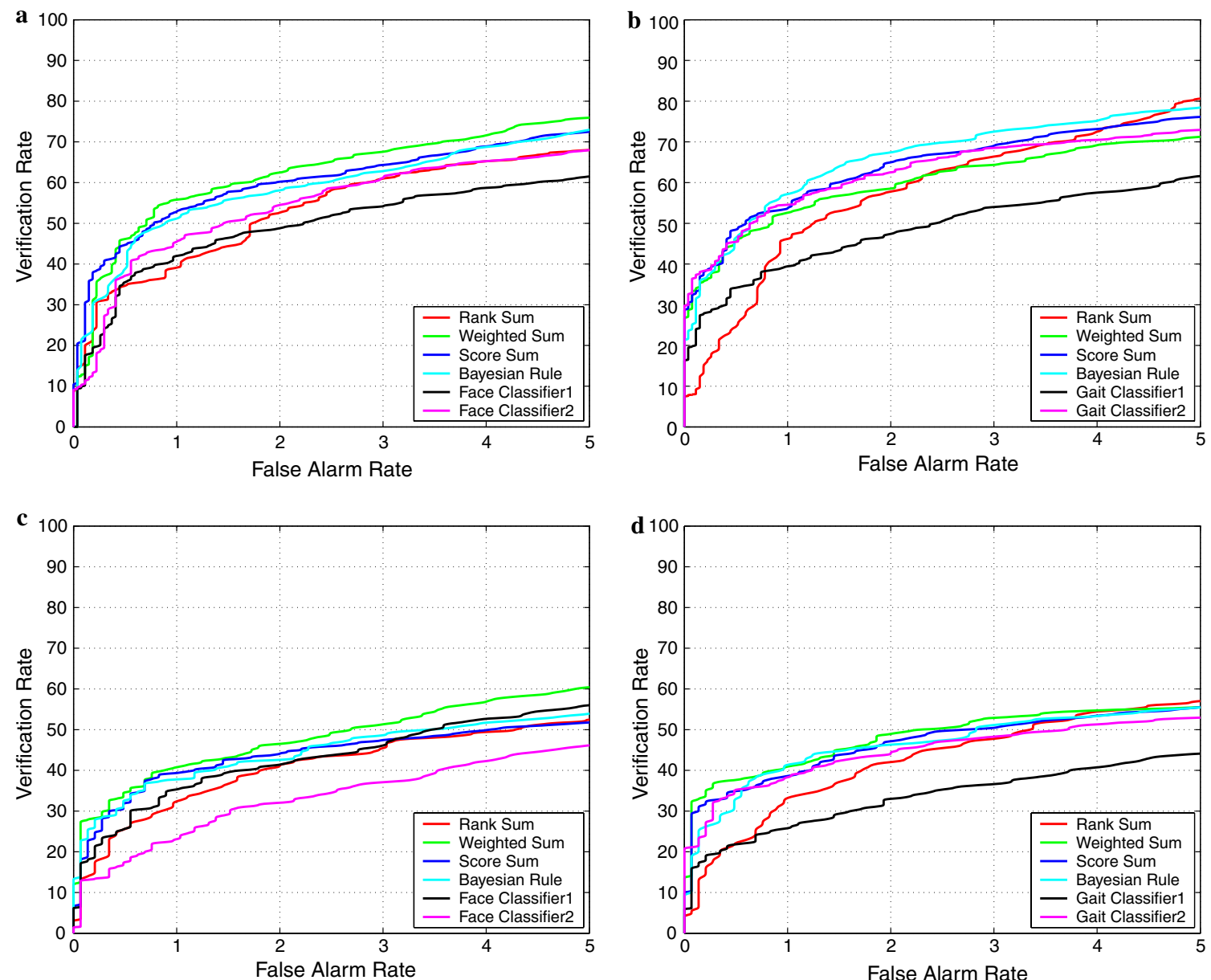

Fig. 16. Performance of intra-modal combination using different strategies. The ROC curves are shown for (a) face + face, same day, $(b)$ gait + gait, same day, (c) face + face, months apart, and (d) gait + gait, months apart. Each plot shows the performance with individual probes and their combinations.
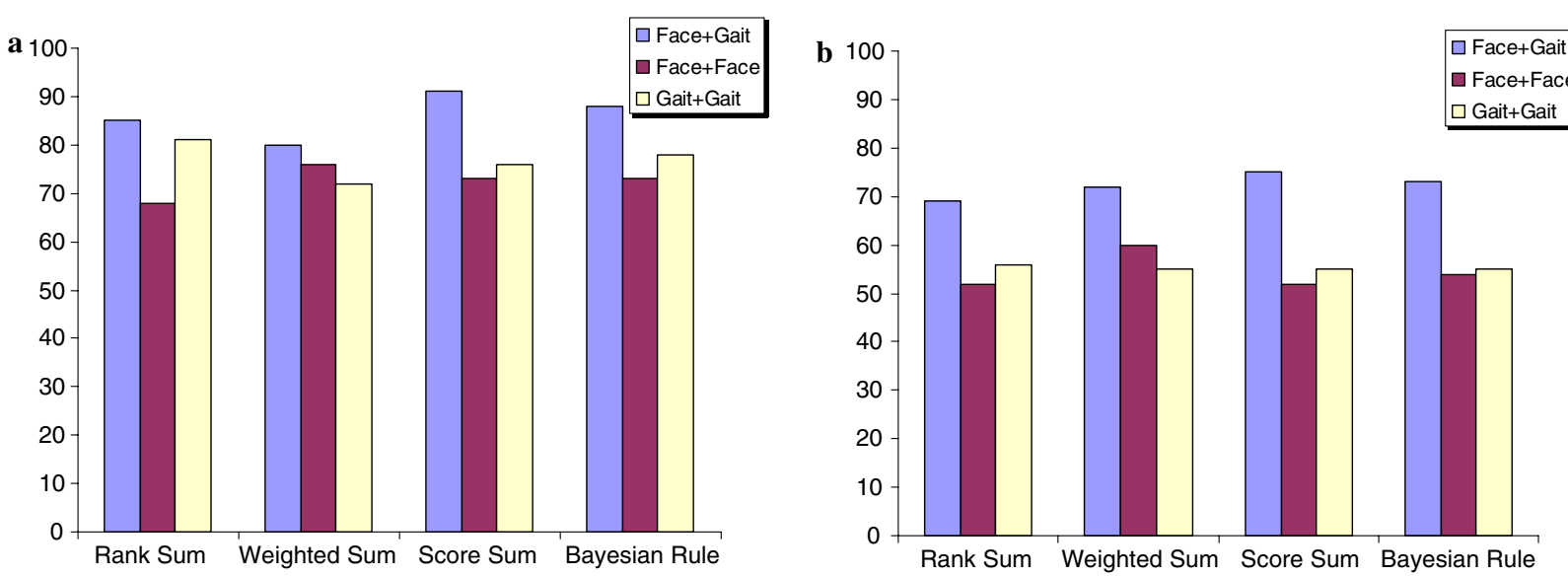

Fig. 17. Bar plot of verification rate at a false alarm rate of $5 \%$ for inter- and intra-modal combination of gait and face for (a) same day data, and (b) data separated by months.

the inter-modal case. Stronger the correlation between the scores, less is the improvement with combination [46]. In fact, this improvement are not only limited to the two hard covariates listed in this paper. In Fig. 18 we compare the performances of all five covariates in our database: view, shoe-type, surface, briefcase, and time, in terms of $P_{V}$ at $5 \%$ false alarm rate. And the results demonstrate the combination substantially improves the recognition. 


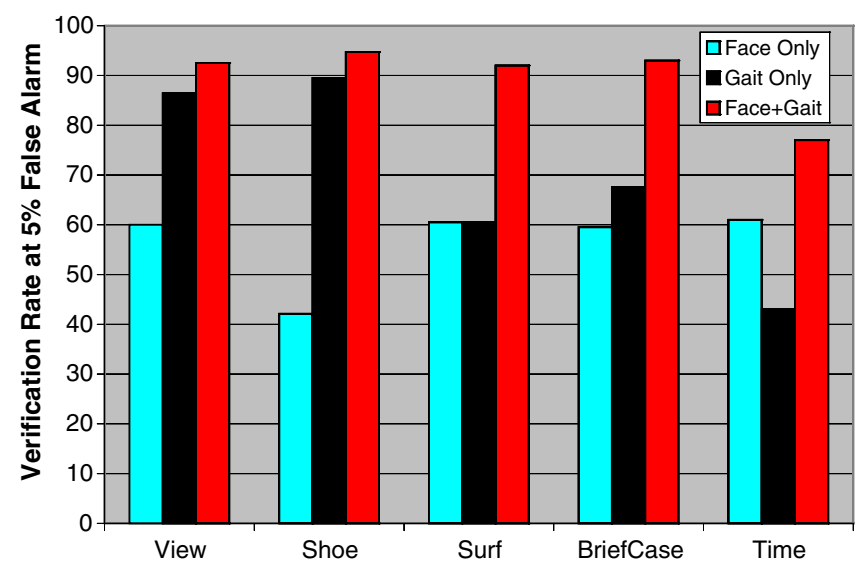

Fig. 18. Bar plot of verification rate at a false alarm rate of $5 \%$ of five covariates in USF HumanID dataset: view, shoe-type, surface, briefcase and time.

\section{Discussion}

\subsection{Underlying reasons of performance improvement from intra-modal combination}

Table 4 lists the number of subjects that are affected by the combination. It lists the number of subjects who were failed to be recognized by each individual modality or both, but were successfully recognized after combination. It also lists the number of subjects who were successfully recognized by one modality or both, but their combination resulted in failure. We see that the performance gained by the combination are mostly from subjects who failed only for one of the two biometrics. The combination helps little for subjects who were not correctly identified by both the individual biometrics. On the other hand, we found that fewer subjects were correctly identified in one classifier but failed after combinations. This is especially true for the score sum combination.

To gain some insight into the nature of the face and gait combination, we plot the decision boundary of the experiment for score sum and Gaussian Bayesian fusion at $5 \%$ false alarm rate in Fig. 19 . The axes are the normalized similarity scores from each modality. We see that (i) the optimal Bayesian decision boundary is roughly linear and is close to the score sum boundary, which explains the high performance with just score sum schemes. And (ii) the non-match scores seem to be uncorrelated forming a nice, symmetric central cluster. This observation would be important for parametric modeling studies. Gaussian models seem to be good for non-match scores.

\subsection{Investigation of gait shape changes with covariates}

We studied the gait changes in terms of silhouette shapes for any particular gait stance. For this, we employ linear discriminant analysis (LDA) of the silhouette shapes of each stance from a set of individuals, with the covariate under study being the "class" or "category" variable. For instance, to study the effect of surface, we place the HMM exemplars of a particular stance from grass in one class and the exemplars for the corresponding stance on concrete in the other class. The leading LDA dimension would be along the direction of maximum discriminability (ratio of between class to within class scatter). It would be interesting to see how different are these directions for different covariates.

Fig. 20 shows these dominant directions (leading eigenvectors of LDA space) as images when comparing silhouettes on grass with shoe type A to (i) sequences on grass with shoe type B, or to (ii) sequences on concrete with shoe type A, or to (iii) sequences on grass with briefcase, or to (iv) sequences on grass taken 6 months apart. The intensity in the images indicates the discriminative body part for the corresponding conditions. All the shown frames are normalized by a same scaling factor for display purposes. For shoe-type change no significant bright areas are seen, indicating that the silhouette shapes in the two classes are similar. For surface change, we find bright spots in the leg portion, particularly in the leg swing phase leg (see image in column 2 and 3 of Fig. 20), suggesting changes in the minimum knee angles due to surface change. In addition, the feet part is highlighted because they are occluded on grass for many subjects. For briefcase carrying, we noticed that the bright spots are concentrated mostly on the trunk part, suggesting that people adjust their upper body stance when carrying weight, which is consistent to our expectations. Finally, for the temporal change, surprisingly, no significant pattern is seen despite the sharp drop in recognition.

Table 4

Number of subject correctly recognized or failed to be recognized by each individual modality or their combination for the same-day data

\begin{tabular}{|c|c|c|c|c|c|c|}
\hline \multirow[t]{2}{*}{ Combination scheme } & \multicolumn{3}{|c|}{$\begin{array}{l}\text { \# failed before combination but succeeded after } \\
\text { combination }\end{array}$} & \multicolumn{3}{|c|}{$\begin{array}{l}\text { \# succeeded before combination but failed after } \\
\text { combination }\end{array}$} \\
\hline & Face only & Gait only & Both & Face only & Gait only & Both \\
\hline Confidence weighted sum & 8 & 12 & 0 & 1 & 4 & 0 \\
\hline Score sum & 14 & 14 & 3 & 2 & 1 & 0 \\
\hline Bayesian rule & 14 & 14 & 3 & 1 & 3 & 0 \\
\hline
\end{tabular}

The total number of subjects is 39 . 

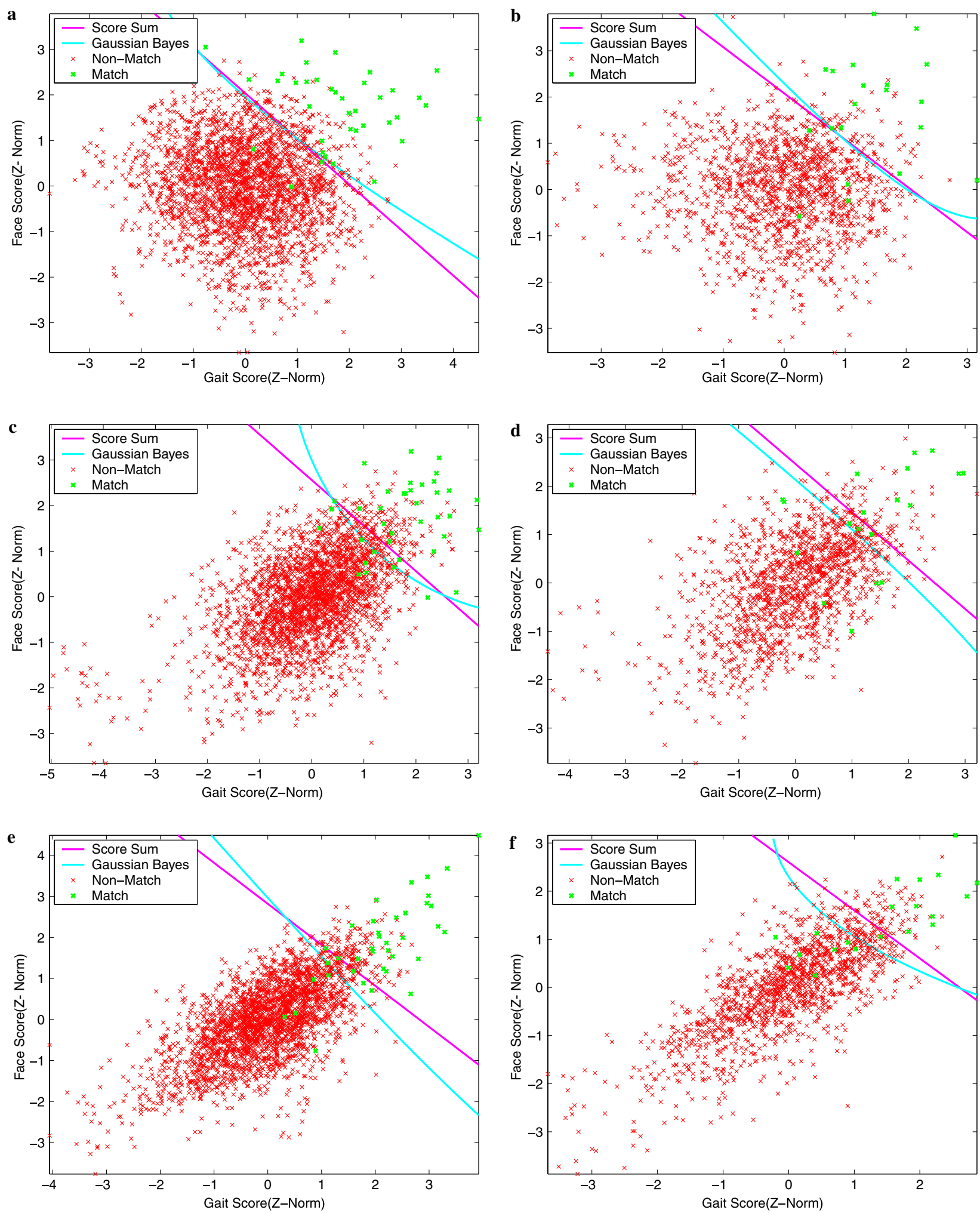

Fig. 19. The decision boundary of the score sum and Bayesian rule combination rules at a false alarm rate of $5 \%$ for (a), (b) face + gait same and different days, (c), (d) face + face same and different days, and (e), (f) gait + gait same and different days, respectively.

\section{Conclusion}

Outdoor face recognition and gait recognition across surface conditions have been found to be hard problems.
In addition, face and gait recognition over time ( $>3$ months apart) is poor. We showed that stance selection can significantly improve gait recognition but not to a level where just gait suffices. We demonstrated that biometric combina- 


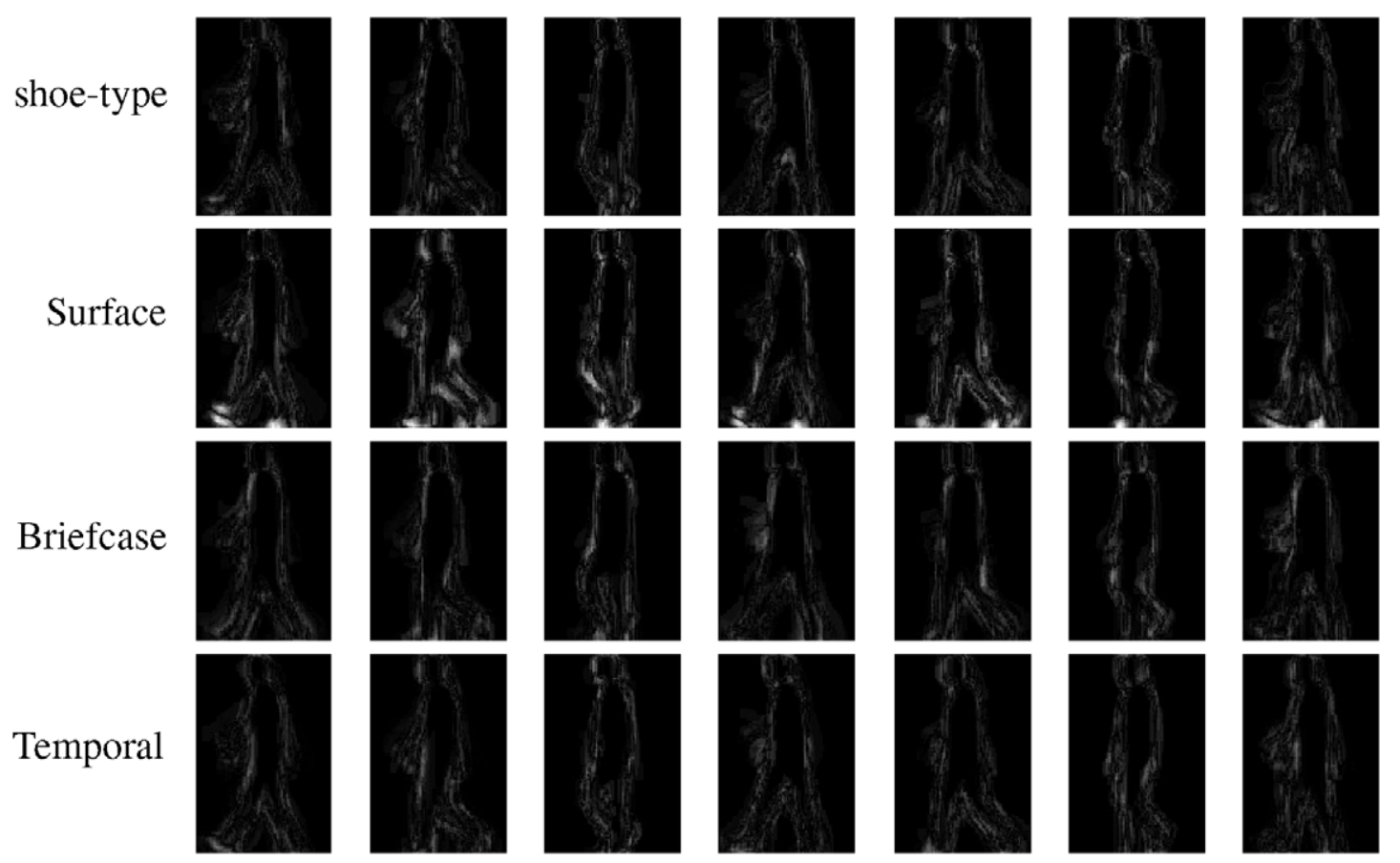

Fig. 20. Samples of the first eigenvectors in LDA space in the changes of shoe-type, surface, and time. The gait phase is column-wise aligned.

tion is an effective strategy for improving performance of these hard biometric problems, involving comparing templates across indoor and outdoors conditions and across months. We find that the score sum rule of combination offer the best performance. We also find the inter-modal combination, i.e. face + gait, is better than not only the individual modalities but also combinations of the same modality, i.e. face + face and gait + gait, which can considered to be sort of statistical control experiments to show improvement of biometric fusion. The inter-modal combination has excellent potential for overcoming the "tough" covariates affecting individual biometrics.

\section{Acknowledgements}

This research was supported by funds from the DARPA Human ID program (F49620-00-1-00388). Ning Yang from Electrical Engineering of USF helped groundtruthing the face images. The code developed by Ross Beveridge et al. at CSU was used to perform face recognition. We also thank Kevin Bowyer and P. Jonathon Phillips for their comments.

\section{References}

[1] B. Achermann, H. Bunke, Combination of face classifiers for person identification, in: International Conference on Pattern Recognition, 1996.

[2] C. BenAbdelkader, R. Cutler, L. Davis, Motion-based recognition of people in eigengait space, in: International Conference on Automatic Face and Gesture Recognition, 2002, pp. 267-272.

[3] A. Bobick, A. Johnson, Gait recognition using static, activity-specific parameters, in: Computer Vision and Pattern Recognition, 2001, pp. $1: 423-430$
[4] D.S. Bolme, Elastic bunch graph matching, Degree of master of science, Colorado State University, 2003.

[5] K. Chang, K.W. Bowyer, S. Sarkar, B. Victor, Comparison and combination of ear and face images in appearance-based biometrics, IEEE Trans. Pattern Anal. Mach. Intel. (2003) 1160-1165.

[6] K.I. Chang, K.W. Bowyer, P.J. Flynn, Face recognition using 2d and 3d facial data, in: Workshop in Multimodal User Authentication, Santa Barbara, California, 2003, pp. 25-32.

[7] K.I. Chang, K.W. Bowyer, P.J. Flynn, Multi-modal 2d and 3d biometrics for face recognition, in: IEEE International Workshop on Analysis and Modeling of Faces and Gestures, Nice, France, 2003.

[8] K.I. Chang, K.W. Bowyer, P.J. Flynn, X. Chen, Multi-biometrics using facial appearance, shape and temperature, in: Sixth IEEE International Conference on Automatic Face and Gesture Recognition, Seoul Korea, May 17-19, 2004.

[9] X. Chen, P.J. Flynn, K.W. Bowyer, Visible-light and infrared face recognition, in: The proceedings of Workshop on Multimodal User Authentication, Santa Barbara, CA, USA, 2003, pp. 48-55.

[10] R. Collins, R. Gross, J. Shi, Silhouette-based human identification from body shape and gait, in: International Conference on Automatic Face and Gesture Recognition, 2002, pp. 366-371.

[11] N. Cuntoor, A. Kale, R. Chellappa, Combining multiple evidences for gait recognition, in: IEEE International Conference on Acoustics, Speech and Signal Processing, 2003.

[12] U. Dieckmann, P. Plankensteiner, T. Wagner, A biometric person identification system using sensor fusion, Pattern Recogn. Lett. (1997) 827-833.

[13] J. Hayfron-Acquah, M. Nixon, J. Carter, Automatic gait recognition by symmetry analysis, in: 3rd International Conference on Audioand Video-Based Biometric Person Authentication, 2001, pp. 272277.

[14] L. Hong, A. Jain, S. Pankanti, Can multibiometrics improve performance? in: IEEE Workshop on Identification of Advanced Technologies, 1999, pp. 59-64.

[15] L. Hong, A.K. Jain, Integrating faces and fingerprints for personal identification, IEEE Trans. Pattern Anal. Mach. Intel. 20 (12) (1998) 1295-1307. 
[16] A.K. Jain, L. Hong, Y. Kulkarni, A multimodal biometric system using fingerprints, face and speech, in: 2nd Int'l Conference on Audioand Video-based Biometric Person Authentication, 1999, pp. 182187.

[17] A.K. Jain, S. Prabhakar, S. Chen, Combining multiple matchers for a high security fingerprint verification system, Pattern Recogn. Lett. 20 (11-13) (1999) 1371-1379.

[18] P. Jonathon Phillips, S. Sarkar, I. Robledo, P. Grother, K. Bowyer, Baseline results for the challenge problem of Human ID using gait analysis, in: International Conference on Automatic Face and Gesture Recognition, 2002, pp. 137-142.

[19] P. Jonathon Phillips, S. Sarkar, I. Robledo, P. Grother, K. Bowyer, The gait identification challenge problem: data sets and baseline algorithm, in: International Conference on Pattern Recognition, 2002, pp. 385-388.

[20] A. Kale, N. Cuntoor, R. Chellappa, A framework for activity specific human identification, in: International Conference on Acoustics, Speech and Signal Processing, 2002.

[21] A. Kale, A. Rajagopalan, N. Cuntoor, V. Kruger, Gait-based recognition of humans using continuous HMMs, in: International Conference on Automatic Face and Gesture Recognition, 2002, pp. 336-341.

[22] A. Kale, A. Roy Chowdhury, R. Chellappa, Fusion of gait and face for human recognition, in: International Conference on Acoustics, Speech, and Signal Processing, 2004.

[23] J. Kittler, M. Hatef, R.P. Duin, J. Matas, On combining classifiers, IEEE Trans. Pattern Anal. Mach. Intel. 20 (3) (1998).

[24] L. Lee, G. Dalley, K. Tieu, Learning pedestrian models for silhouette refinement, in: International Conference on Computer Vision, 2003.

[25] L. Lee, W. Grimson, Gait analysis for recognition and classification, in: International Conference on Automatic Face and Gesture Recognition, 2002, pp. 155-162.

[26] J. Little, J. Boyd, Recognizing people by their gait: the shape of motion, Videre 1 (2) (1998) 1-33.

[27] Z. Liu, L. Malave, A. Osuntogun, P. Sudhakar, S. Sarkar, Toward understanding the limits of gait recognition, in: SPIE Processings of Defense and Security Symposium: Biometric Technology for Human Identification, 2004.

[28] Z. Liu, L. Malave, S. Sarkar, Studies on silhouette quality and gait recognition, in: Computer Vision and Pattern Recognition, vol. II, 2004, pp. 704-711.

[30] Z. Liu, S. Sarkar, Effect of silhouette quality on hard problems in gait recognition, IEEE Trans. Systems, Man, and Cybernetics (Part B) 35 (2) (2005) 170-183.

[31] X. Lu, Y. Wang, A.K. Jain, Combining classifiers for face recognition, in: IEEE International Conference on Multimedia And Expo, vol. 3, 2003, pp. 13-16.

[32] O. Melnik, Y. Vardi, C. Zhang, Mixed group ranks: Preference and confidence in classifier combination, Rutgers Statistics Department Tech Report, 2003.

[33] S. Niyogi, E. Adelson, Analyzing gait with spatiotemporal surfaces, Computer Vision and Pattern Recognition (1994).

[34] P.J. Phillips, P. Grother, R.J. Micheals, D.M. Blackburn, E. Tabassi, M. Bone, Face recognition vendor test 2002, http://www.frvt.org, March 2002.

[35] P.J. Phillips, H. Moon, S.A. Rizvi, P.J. Rauss, The FERET evaluation methodology for face-recognition algorithms, IEEE Trans. Pattern Anal. Mach. Intel. 22 (10) (2000).
[36] S. Prabhakar, A.K. Jain, Decision-level fusion in fingerprint verification, Pattern Recogn. 35 (4) (2002) 861-874.

[37] L. Rabiner, B.H. Juang, Fundamental of Speech Recognition, Prentice Hall, 1993.

[38] I. Robledo Vega, S. Sarkar, Representation of the evolution of feature relationship statistics: human gait-based recognition, IEEE Trans. Pattern Anal Mach. Intel, to appear.

[39] A. Ross, A.K. Jain, Information fusion in biometrics, Pattern Recogn. Lett. 24 (2003) 2115-2125.

[40] B. Schiele, How many classifiers do i need?, in: International Conference on Pattern Recognition, 2002, pp. II: 176-179.

[41] G. Shakhnarovich, T. Darrell, On probabilistic combination of face and gait cues for identification, in: Proceedings of the Fifth IEEE International Conference on Automatic Face and Gesture Recognition, 2002.

[42] G. Shakhnarovich, L. Lee, T. Darrell, Integrated face and gait recognition with multiple views, Computer Vision Pattern Recogn. (2001).

[43] J. Shutler, M. Nixon, C. Carter, Statistical gait description via temporal moments, in: 4th IEEE Southwest Symposium on Image Analysis and Interference, 2000, pp. 291-295.

[44] A. Sundaresan, A.R. Chowdhury, R. Chellappa, A hidden markov model based framework for recognition of humans from gait sequences, in: IEEE International Conference on Image Processing, 2003.

[45] R. Tanawongsuwan, A. Bobick, Gait recognition from time-normalized joint-angle trajectories in the walking plane, in: Computer Vision and Pattern Recognition, 2001, pp. 11:726-731.

[46] D.M. Tax, M. van Breukelen, R.P. Duin, J. Kittler, Combining multiple classifiers by averaging or by multiplying? Pattern Recogn. 33 (9) (2000) 1475-1485.

[47] D. Tolliver, R. Collins, Gait shape estimation for identification, in: 3rd International Conference on Audio- and Video-Based Biometric Person Authentication, 2003.

[48] A. Veeraraghavan, A.R. Chowdhury, R. Chellappa, Role of shape and kinematics in human movement analysis, in: Computer Vision and Pattern Recognition, Washington D.C., USA, 2004.

[49] L. Wang, W. Hu, T. Tan, A new attempt to gait-based human identification, in: International Conference on Pattern Recognition, vol. 1, 2002, pp. 115-118.

[50] L. Wang, T. Tan, H. Ning, W. Hu, Silhouette analysis-based gait recognition for human identification, IEEE Trans. Pattern Anal. Mach. Intel. 25 (2003) 1505-1518.

[51] Y. Wang, T. Tan, A.K. Jain, Combining face and iris biometrics for identity verification, in: International Conference on Audio- and Video-Based Biometric Person Authentication, 2003, pp. 805-813.

[52] L. Wiskott, J.-M. Fellous, N. Kruger, C. von der Malsburg, Face recognition by elastic bunch graph matching, IEEE Trans. Pattern Anal. Mach. Intel. 19 (7) (1997) 775-779.

[53] K. Woods, W. Philip Kegelmeyer Jr., K. Bowyer, Combination of multiple classifiers using local accuracy estimates, IEEE Trans. Pattern Anal. Mach. Intel. 19 (4) (1997).

[54] J. Zhou, D. Zhang, Face recognition by combining several algorithms, in: International Conference on Pattern Recognition, 2002, pp. III: $497-500$

[55] Y. Zuev, S. Ivanon, The voting as a way to increase the decision reliability, Found. Inf./Decis. Fusion Appl. Eng. Probl. (1996) 206210 . 